\title{
Discussion of Ways to Energy-Filter the Electron Backscattering Pattern (EBSP) in the Scanning Electron Microscope (SEM).
}

Oliver C. Wells, Research Staff Member Emeritus, IBM Research Division, PO Box 218, Yorktown Heights, NY 10598

An electron backscattering pattern (EBSP) is formed by the electrons that are scattered with the highest energies from a crystalline target that is illuminated with an electron beam (EB) of kiloelectron-volt (keV) energy. In appearance it closely resembles the electron channeling pattern (ECP) that is formed in the scanning electron microscope (SEM) when the incident EB is rocked about a point. According to one possible explanation, it is assumed that in either case the pattern is formed by the electrons that leave the specimen with a minimum loss of energy in the reflected electron peak following a single wide-angle Rutherford-type scattering event (typically through about a right angle) close to the surface. Now if ECP and EBSP are reciprocal, they can be related by reversing the direction of the (zero-loss) electrons in the specimen. It can then be argued that these patterns must be formed because the probability of such a Rutherford-type wide-angle scattering event is modulated by both the incoming (ECP) and outgoing (EBSP) channeling conditions [1-6]. The slower scattered electrons undergo scattering, channeling and diffraction events that are in addition to this basic process. The related reflection high-energy electron diffraction (RHEED) pattern is formed with grazing incident and exit directions with electrons that are diffracted by Bragg planes parallel to the surface.

Generally the EBSP is formed over a limited solid angle by placing a detecting screen in line-ofsight from the specimen or (in principle) with a retarding-field energy filter [6]. An alternative approach that is suggested here is to magnetically filter the scattered electrons by mounting the sample between the polepieces of a magnetic immersion lens, with the limitations: (a) The sample must be totally non-magnetic. (b) It will be irradiated more heavily than with the standard EBSP.

Fig. 1 shows a solid sample mounted between the polepieces of a magnetic immersion lens. The magnetic field is adjusted so as to focus the incident EB onto the specimen. This same magnetic field also deflects the scattered electrons to follow spiral paths that periodically return to the lens axis. The fastest scattered electrons (reflected electron peak) can reach a limiting surface at the end of the first half-turn that cannot be reached by the slower scattered electrons. The slower scattered electrons can, however, go beyond this limiting surface on subsequent turns of the spiral path if not prevented from doing so [7]. It has been demonstrated that a detector that is placed at a short distance inside this limiting surface will collect only the fastest scattered electrons and can be used to give a magnetically filtered low-loss electron (LLE) image in the SEM [8-10].

The next step is to make use of this situation to give an energy-filtered EBSP, based on the idea that there is a one-to-one correspondence between the direction in which the fastest scattered electrons leave the specimen and the point at which these electrons reach (just touch) the limiting surface:

(a) Thus, if a curved detecting screen can be placed at exactly this boundary surface over a sufficiently large area, then it will show an energy-filtered EBSP by detecting the fastest scattered electrons as they just touch this surface. However, it might not be easy to align an image screen that must be curved in two directions with the limiting surface that will move as the incident EB is moved on the specimen. 
(b) Another method would be to place a flat image-forming screen (similar to that in a digital camera) so that it intersects the limiting surface at an angle. The image is then analysed by a computer to locate the single line of pixels that lies along the edge of the illuminated area at the boundary. The sample is then either rotated about some axis or the screen is moved in some way to give a series of lines of pixels that can be placed side by side to form an EBSP. The incident EB can be scanned and/or deflected under computer-control so as to always land at the same point on the specimen as it rotates (for example) about the axis of the lens. (The idea of rotating the sample and merging the relevant information as the image changes can in principle also be applied to the conventional method for recording EBSP.) It is believed that these techniques should be investigated for the purpose of recording an energy-filtered EBSP [11].

\section{References:}

[1] L. Reimer, Scanning Electron Microscopy, Physics of Image Formation and Microanalysis, Springer-Verlag, 1985.

[2] L. Reimer, Private communication: "...scattering is more likely to occur into a favorable direction than into an unfavorable one..." 1994.

[3] D.C. Joy, Proc. 52 $2^{\text {nd }}$. Ann. Mtg. MSA, (1994) 592.

[4] O.C. Wells, Scanning 21 (1999) 368.

[5] ECP with a retarding-field energy filter: E.D. Wolf, P.J. Coane and T.E. Everhart, Cong. Int. Micro. Electron., Grenoble, 2, (1970) 595.

[6] RHEED pattern with a retarding-field energy filter: staib-us@ staib-instruments.com

[7] O.C. Wells and E. Munro, Ultramicroscopy 47 (1992) 101.

[8] O.C. Wells, F.K. LeGoues and R.T. Hodgson, Appl. Phys. Lett. 56 (1990) 2351.

[9] O.C. Wells, E. Munro and I.M. Fisher, Inst. Phys. Conf. Series No. 119, Section 5 (EMAG91), (1991) 181.

[10] O.C. Wells, Proc. ALC'01 (Nara, Japan, Nov. 2001) in press, 2002.

[11] I would like to thank P.E. Batson, J. Del Vecchio, D.J. Dingley, L. Dylla, R. von Gutfeld, M. Kammler, D.E. Newbury, J.R. Michael, M.T. Postek and R.M. Tromp for helpful discussions.

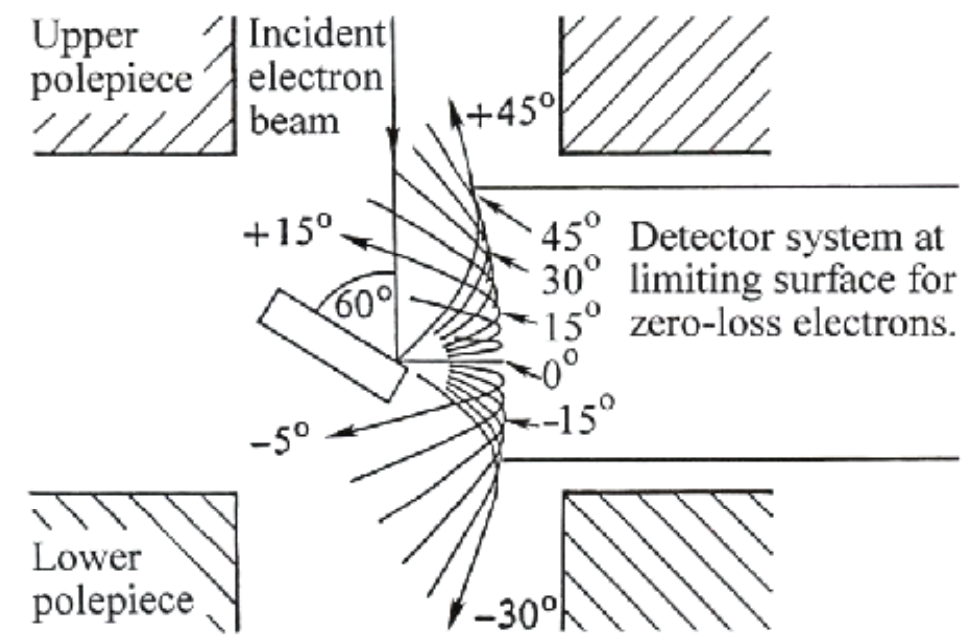

FIG. 1. Sample mounted between the polepieces of a magnetic immersion lens, showing the limiting surface just reached by the fastest electrons that are scattered from the sample at the angles shown above or below the horizontal plane ( $\mathrm{r}-\mathrm{z}$ plots of trajectories; modified from ref. 10). 